MATEMATIKA, 2017, Volume 33, Number 1, 105-111

(C) Penerbit UTM Press. All rights reserved

\title{
Geometries, independence spaces and infinite antimatroids
}

\author{
Hua Mao \\ Department of Mathematics, Hebei University, \\ Baoding 071002, China \\ e-mail: yushengmao@263.net
}

\begin{abstract}
This paper generalizes the notion of antimatroids from the case that the ground set $X$ is a finite set to the case that $X$ is an arbitrary set. It not only deals with the relationships between geometries and independence spaces, but also demonstrates the relationships between convex geometries and arbitrary antimatroids.
\end{abstract}

Keywords geometry; closed set; independence space; infinite antimatroid

2010 Mathematics Subject Classification 05B35; 52A99

\section{Introduction and Preliminaries}

The infinite matroid theory which has been most fruitfully used seems to be independence space which is one of the more frequently studied classes of infinite matroids([1-7]). However, to our knowledge, few authors have indicated the links between independence spaces and geometries. Besides, as an important combinatorial structure, antimatroid has attracted the attention of many researchers and yields many research reports ([8-16]). It has been extended to infinite sets in different ways (cf.[12-15]).

The purpose of this paper is to discuss the main lines taken by research into geometry and independence space and present the definition of infinite antimatroids by a geometric way. The definition of infinite antimatroids here is different from the others we have known (cf.[12,13] and the references therein). Simultaneously, it indicates the links between convex geometry and infinite antimatroids. The results of this paper will build a foundation for the realization of solving the problems on independence spaces and infinite antimatroids with the use of geometry theory and convex geometry theory respectively.

Initially, we will recall and present some basic knowledge. In what follows, we work over a ground-possibly infinite-set $E$. $2^{S}$ denotes the set of all subsets of a set $S ; X \subset \subset Y \subseteq E$ indicates that $X$ is a finite subset of $Y$. A set system over $E$ is a non-empty family contained in $2^{E}$. That $(E, \mathcal{F})$ is accessible means that for every nonempty set $X \in \mathcal{F}$, there exists some $x \in X$ such that $X \backslash x \in \mathcal{F}$.

\section{Definition 1}

(1) $[1$, p.387\&2,p.74] An independence space $M(E)$ is a set $E$ together with a collection $\mathcal{I} \subseteq 2^{E}$ (called independent sets) such that

(i1) $\mathcal{I} \neq \emptyset$;

(i2) A subset of an independent set is independent;

(i3) If $I_{1}, I_{2} \in \mathcal{I}$ and $I_{j} \subset \subset E(j=1,2)$ with $\left|I_{1}\right|<\left|I_{2}\right|$, then $\exists x \in I_{2} \backslash I_{1}$ satisfies $I_{1} \cup x \in \mathcal{I}$

(i4) If $X \subseteq E$ and every finite subset of $X$ is in $\mathcal{I}$, then $X$ is in $\mathcal{I}$.

$A$ subset $X$ of $E$ is dependent if $X \notin \mathcal{I}$. A circuit of $M(E)$ is a minimal dependent set. 
(2) [1,p.388] We define the closure operator of $M(E)$ by $x \in \sigma(A)$ if $x \in A$ or if there exists a circuit $C$ with $x \in C \subseteq A \cup x$. A set $X$ is closed of $M(E)$ if $\sigma(X)=X$.

Lemma 1 [1,p.388] A function $\sigma: 2^{E} \rightarrow 2^{E}$ is the closure operator of an independence space $M(E)$ if and only if for $X, Y$ subsets of $E$, and $x, y \in E$

(s1) $X \subseteq \sigma(X)$;

(s2) $Y \subseteq X$ implies $\sigma(Y) \subseteq \sigma(X)$;

(s3) $\sigma(X)=\sigma(\sigma(X))$;

(s4) If $y \notin \sigma(X), y \in \sigma(X \cup x)$, then $x \in \sigma(X \cup y)$;

(s5) If $a \in \sigma(X)$ for some $X \subseteq E$, then $a \in \sigma\left(X_{f}\right)$ for some $X_{f} \subset \subset X$.

Definition 2 [17,p.240] A geometry $\left(A,^{-}\right)$is a set $A$ and a function $X \mapsto \bar{X}$ of $2^{A}$ into itself satisfying the following conditions:

(i) - is a closure relation, that is,

(i $\left.i_{1}\right) X \subseteq \bar{X}$

$\left(i_{2}\right)$ If $\bar{X} \subseteq Y$, then $\bar{X} \subseteq \bar{Y}$;

$\left(i_{3}\right) \bar{X}=\bar{X}$.

(ii) $\bar{\emptyset}=\emptyset$, and $\overline{\{x\}}=\{x\}$ for $x \in A$;

(iii) If $x \in \overline{X \cup y}$, but $x \notin \bar{X}$, then $y \in \overline{X \cup x}$;

(iv) If $x \in \bar{X}$, then $x \in \overline{X_{1}}$ for some $X_{1} \subset \subset X$.

\section{Remark 1}

(1) Gräzter points out in $[17, p .240]$ that the (i) in Definition 2 means that $\left(A,^{-}\right)$is a closure space. A subset $X$ of a closure space is called closed if and only if $\bar{X}=X$. It follows simply form $\left(i_{1}\right)-\left(i_{3}\right)$, that $\bar{X}$ is the smallest closed set containing $X$.

(2) Let $M(E)$ be an independence space. An element $x$ of $E$ is a loop if $\{x\}$ is a dependent set; two elements $x, y$ of $E$ are parallel if they are not loops but $\{x, y\}$ is a dependent set. $M(E)$ is simple if it has no loops or parallel elements.

(3) In this paper, a general geometry $\left(A,^{-}\right)$is a set $A$ and a function $X \mapsto \bar{X}$ of $2^{A}$ onto itself satisfying the conditions (i), (iii) and (iv) in Definition 2.

(4) As the finite case (cf.[8,9]), let $\mathcal{F} \subseteq 2^{E}$ be a set system, and we can that a basis of a subset $A \subseteq E$ of $(E, \mathcal{F})$ is a (inclusion-wise) maximal element in $\mathcal{F}$ of $A$; a loop is an element of $E$ that is contained in no basis; $\mathcal{F}$ is normal if it does not contain loops.

(5) All the knowledge of poset arise from [17]. As the authors in [8,p.5] and [9,p.286] pointed out, all of the antimatroids in $[8,9]$ are finite.

We extend some concepts linked to finite antimatroids (cf. [8,9]) to infinite cases.

\section{Definition 3}

(1) A function $\tau: 2^{E} \rightarrow 2^{E}$ is a closure operator if $\tau$ satisfies the following conditions for any $A, B \subseteq E$ :

(co1) $\tau(\emptyset)=\emptyset$;

(co2) $A \subseteq \tau(A)$;

(co3) $A \subseteq B$ implies $\tau(A) \subseteq \tau(B)$;

$(\operatorname{co} 4) \tau(\tau(A))=\tau(A)$.

(2) We call $E$ endowed with a closure operator $\tau$ a convex geometry if the following axiom is satisfied:

(AE) If $y, z \notin \tau(X)$ and $z \in \tau(X \cup y)$, then $y \notin \tau(X \cup z)$.

(3) A convex geometry $(E, \tau)$ is called infinite convex geometry if $(E, \tau)$ satisfies 
(AC) For any closed sets of $(E, \tau)($ i.e. $\tau(X)=X$ for $X \subseteq E) A$ and $B$, if $B \subset A$, then there exists $a \in A \backslash B$ satisfying $A \backslash a$ is closed.

(4) Let $\mathcal{F} \subseteq 2^{E}$ be a set system with $\emptyset \in \mathcal{F}$. An infinite antimatroid is a pair $(E, \mathcal{F})$ with $\mathcal{F}$ (called feasible sets)satisfying the following conditions:

(I) $\mathcal{F}$ is normal;

(II) $\mathcal{F}$ is closed under union, i.e. $X_{\alpha} \in \mathcal{F}(\alpha \in \mathcal{A})$ implies $\bigcup_{\alpha \in \mathcal{A}} X_{\alpha} \in \mathcal{F}$;

(III) For $X, Y \in \mathcal{F}, Y \subset X$, there is an $x \in X \backslash Y$ such that $Y \cup x \in \mathcal{F}$.

From the definition of infinite antimatroids, we easily progress to the following:

Corollary 1 Every subset in an infinite antimatroid has a unique basis.

\section{Remark 2}

(1) By Lemma 1, we denote an independence space $(E, \mathcal{I})$ as $(E, \sigma)$.

(2) Let $\mathcal{F}$ be a set system and $\mathcal{N}$ be the complement of $\mathcal{F}$, i.e. $\mathcal{N}=\{X: E \backslash X \in \mathcal{F}\}$. Then $\mathcal{N}$ gives rise to the following operator $\mu(A)=\cap\{X: A \subseteq X, X \in \mathcal{N}\}$ for $A \subseteq E$. We call $\mu$ the relative operator to $\mathcal{N}$ and $\mathcal{N}$ the relative to $\mu$.

(3) Let $(E, \tau)$ be a convex geometry and $\mathcal{N}$ the family of all closed sets of $(E, \tau)$.

(i) We assert that $\mathcal{N}$ is closed under intersection, i.e. $X_{\alpha} \in \mathcal{N}(\alpha \in \mathcal{A})$ means $\bigcap_{\alpha \in \mathcal{A}} X_{\alpha} \in \mathcal{N}$. The sketch of this proof is as follows:

By (co1)-(co4), one has $\emptyset \in \mathcal{N}$ and $E \in \mathcal{N}$. Since $\tau\left(\bigcap_{\alpha \in \mathcal{A}} X_{\alpha}\right) \subseteq \bigcap_{\alpha \in \mathcal{A}} \tau\left(X_{\alpha}\right) \subseteq$ $\tau\left(\bigcap_{\alpha \in \mathcal{A}} \tau\left(X_{\alpha}\right)\right)$ in view of (co2) and (co3), besides, $\tau\left(\bigcap_{\alpha \in \mathcal{A}} X_{\alpha}\right) \subseteq \tau\left(\bigcap_{\alpha \in \mathcal{A}} \tau\left(X_{\alpha}\right)\right) \subseteq$ $\tau\left(\bigcap_{\alpha \in \mathcal{A}} X_{\alpha}\right)$ by (co4) and $X_{\alpha} \in \mathcal{N}(\alpha \in \mathcal{A})$, one has $\tau\left(\bigcap_{\alpha \in \mathcal{A}} X_{\alpha}\right)=\bigcap_{\alpha \in \mathcal{A}} \tau\left(X_{\alpha}\right)=$ $\bigcap_{\alpha \in \mathcal{A}} X_{\alpha}$, i.e. $\bigcap_{\alpha \in \mathcal{A}} X_{\alpha} \in \mathcal{N}$.

Based on this result, one gets $\tau(A)=\cap\{X \in \mathcal{N}: A \subseteq X\}$, namely, $\tau$ is relative to $\mathcal{N}$.

(ii) Conversely, if $\mathcal{N} \subseteq 2^{E}$ is a set system preserved under intersection, then $\mu(A)=$ $\cap\{C \in \mathcal{N}: A \subseteq C\}$ is a closure operator, and this gives a one-to-one correspondence between closure operators and intersection invariant set systems containing $E$. In particular, a closure operator can be specified by giving the family $\mathcal{N}$ of closed sets.

(4) We notice that actually, by the above $(3)$, if $(E, \tau)$ is a convex geometry, then $\tau$ is only relative to the family of all closed sets of $(E, \tau)$, that is, if $\tau$ is relative to a set system $\mathcal{N}$, then $\mathcal{N}$ must be the family of all closed sets of $(E, \tau)$.

(5) Suppose $\mathcal{F}$ is a normal set system and every member in $\mathcal{F}$ is finite. If $\mathcal{F}$ is accessible and closed under union, then similarly to the proof in [9,Proposition 8.2.7,p.291], we have $\emptyset \in \mathcal{F}$, and (III) in Definition 3 holds. That is to say, under the assumption of $\mathcal{F}$ above, (III) is redundant for the definition of an infinite antimatroid.

This also tells us that the definition of an infinite antimatroid is indeed the generalization of that of finite antimatroids(cf. $[8,9])$.

Corollary 2 Let $\mathcal{F}$ be a set system such that $X \in \mathcal{F}$ implies $X \subset \subset \subset$. Let $\mathcal{N}$ be the complement of $\mathcal{F}$ and $\tau$ the relative operator of $\mathcal{N}$.

(1) $\mathcal{F}$ is an infinite antimatroid if and only if $\mathcal{F}$ is accessible and satisfying (I) and (II).

(2) $(E, \tau)$ is a convex geometry if and only if $\tau$ satisfies (co1)-(co4) and (AE). 


\section{Proof}

(1) Routine verification from Definition 3 and Remark 2.

(2) $(\Rightarrow)$ It is easy to get by Definition 3 .

$(\Leftarrow) \tau$ satisfies $(\mathrm{co} 1)-(\mathrm{co} 4)$ and $(\mathrm{AE})$ and $\mathcal{F}$ is the complement of $\mathcal{N}$. By (co1) and (co4), $\emptyset \in \mathcal{F}$ and $E \in \mathcal{F}$. Next we show that $(E, \mathcal{F})$ is accessible. Let $X \in \mathcal{F}$ and $x \in X$ satisfy that $\tau(E \backslash(X \backslash x))$ is minimal with respect to set theoretic containment. Suppose $X \backslash x \notin \mathcal{F}$. By (co2), there exists an element $x^{\prime} \in \tau(E \backslash(X \backslash x)) \backslash(E \backslash(X \backslash x)) \subseteq$ $X \backslash x$. Then $E \backslash\left(X \backslash x^{\prime}\right) \subseteq \tau(E \backslash(X \backslash x))$, hence by (co3) and (co4), $\tau\left(E \backslash\left(X \backslash x^{\prime}\right)\right) \subseteq$ $\tau(E \backslash(X \backslash x))$, where the inclusion is strict, since, by (AE), $x \notin \tau\left(E \backslash\left(X \backslash x^{\prime}\right)\right)$, a contradiction to the choice of $x$. By Remark $2, \mathcal{N}$ is closed under intersection, and in view of (1), $\mathcal{F}$ satisfies (III), and so, we get $\tau$ satisfying (AC).

Let $(E, \tau)$ be an infinite convex geometry and $\mathcal{N}$ the family of all the closed sets of $(E, \tau)$. Suppose $\mathcal{N}$ satisfies that for any $X \in \mathcal{N}$, it implies $E \backslash X$ is finite. Then by Corollary 2, we see that the condition (AC) is redundant because it could be deduced from (co1)-(co4) with (AE). This result tells us that the definition of infinite convex geometry presented here is indeed the generalization of that relative to finite convex geometry (cf. $[8,9])$.

\section{Relations}

This section deals with the relationships between geometries and independence spaces. In addition, it shows the relationships between convex geometries and infinite antimatroids.

\section{Theorem 1}

(1) The correspondence between a geometry $\left(E,^{-}\right)$and the simple independence space $(E, \sigma)$, where $\sigma$ is just ${ }^{-}$, is a bijection between the set of geometries and the set of simple independence spaces.

(2) A set $E$ and a function $X \mapsto \bar{X}$ of $2^{E}$ unto itself is a general geometry if and only if $\left(E,,^{-}\right)$is an independence space.

Proof Routine verification.

Let $E$ be the set of edges of a graph. The edge geometry (cf.[17,p.244]) is $\left(E,^{-}\right)$, then by $\left[17\right.$,Theorem 12,p.244], $\left(E,^{-}\right)$is a geometry. Hence by Theorem 1 , the edge geometry is a simple independence space. This instance tells us that Theorem 1 will play a role in discussing the properties of a geometry, and certainly vice versa.

Lemma 2 Let $(E, \mathcal{F})$ be an infinite antimatroid, $\mathcal{N}=\{X: E \backslash X \in \mathcal{F}\}$ and $\tau$ the relative operator to $\mathcal{N}$. Then $(E, \tau)$ is a convex geometry and an infinite convex geometry.

Proof Since the feasible sets of $(E, \mathcal{F})$ are closed under union, and further, the system of complements $\mathcal{N}$ is closed under intersection, by Remark 2, $\tau(A)=\cap\{X \in \mathcal{N}: A \subseteq X\}$ for $A \subseteq E$, and hence this induces a closure operator $\tau$. That is, it is straightforward to check that $\tau$ satisfies (co1)-(co4). We only have to prove the property (AE).

For this purpose, let $X \subseteq E, y, z \notin \tau(X), z \in \tau(X \cup y)$, and let $B$ be the basis of $E \backslash X$ and $A$ the basis of $E \backslash(X \cup y)$ in $\mathcal{F}$. Hence, by Corollary $1, A, B$ is the unique basis of $E \backslash(X \cup y)$ and $E \backslash X$ respectively. Since $y, z \notin \tau(X)=\cap\{T \in \mathcal{N}: X \subseteq T\}=\cap\{E \backslash T \in \mathcal{F}:$ $E \backslash X \supseteq E \backslash T\}$ implies $y, z \notin X$, otherwise, $y, z \in \tau(X)$, a contradiction. Hence $y, z \in E \backslash X$. Because $(E, \mathcal{F})$ is an infinite antimatroid, one has that both $y$ and $z$ are contained in a basis of $E \backslash X$, that is, $y, z \in B$. We know $z \in \tau(X \cup y)=\cap\{D \in \mathcal{N}: X \cup y \subseteq D\}$. 
Suppose $z \in A \subseteq E \backslash(X \cup y)$. Then this means $X \cup y \subseteq E \backslash A \in \mathcal{N}$ and $z \notin E \backslash A$. But $z \in \tau(X \cup y)$ and $X \cup y \subseteq E \backslash A \in \mathcal{N}$ together tell us $z \in E \backslash A$, a contradiction to the supposition. Thus $z \notin A$. In addition, by the choice of $A$ and $B$ and the above discussion, $A \subseteq B \backslash\{y, z\}$. So we can augment $A$ to some set $A \cup x \in \mathcal{F}$, where $x \in B$. Since $A$ is a basis of $E \backslash(X \cup y)$, we must have $x=y$, i.e. $A \cup y$ is a feasible subset of $E \backslash(X \cup z)$ and $y \notin \tau(X \cup z)$.

Up till now, we see that $(E, \tau)$ is a convex geometry. By (III) in Definition 3 and the complement of $\mathcal{N}$ for $\mathcal{F}, \tau$ satisfies $(\mathrm{AC})$ obviously. Therefore, $(E, \tau)$ is an infinite convex geometry.

Lemma 3 Let $(E, \tau)$ be a convex geometry and $\mathcal{N}$ the family of closed sets of $(E, \tau)$.

(1) For all closed sets $A \subset B$, there exists $x \in B \backslash A$ such that $A \cup x$ is closed.

(2) Let $\mathcal{F}$ be the complement of $\mathcal{N}$. Then $\mathcal{F}$ is normal and closed under union.

\section{Proof}

(1) Suppose $C$ is a minimal closed set such that $A \subset C \subseteq B$, and let $x \in C \backslash A$. Because, if $y \in \tau(A \cup x) \backslash(A \cup x)$, then by the anti-exchange condition (AE), $x \notin \tau(A \cup y)$; hence $A \subset \tau(A \cup y) \subset C$ which contradicts the minimality of $C$. So, there is $x \in C \backslash A \subseteq B \backslash A$ such that $A \cup x$ is closed.

(2) Since $\tau(\emptyset)=\emptyset$, i.e. $\tau(E \backslash E)=E \backslash E$, which implies $E \in \mathcal{F}$. Hence $\mathcal{F}$ is normal.

Finally, let $X_{\alpha} \in \mathcal{F}(\alpha \in \mathcal{A})$. Then the properties of (co1)-(co4) yield $E \backslash \bigcup_{\alpha \in \mathcal{A}} X_{\alpha} \subseteq$ $\tau\left(E \backslash \bigcup_{\alpha \in \mathcal{A}} X_{\alpha}\right) \subseteq \bigcap_{\alpha \in \mathcal{A}} \tau\left(E \backslash X_{\alpha}\right)=\bigcap_{\alpha \in \mathcal{A}}\left(E \backslash X_{\alpha}\right)=E \backslash \bigcup_{\alpha \in \mathcal{A}} X_{\alpha}$. Hence $\bigcup_{\alpha \in \mathcal{A}} X_{\alpha} \in \mathcal{F} . \square$

Lemma 4 Let $\mathcal{N}$ be the family of closed sets of a convex geometry $(E, \tau)$ such that $X \in \mathcal{N}$ implies $E \backslash X \subset \subset E$. Then $(E, \mathcal{F}=\{X: E \backslash X \in \mathcal{N}\})$ is an infinite antimatroid.

Proof According to Lemma 3 and Definition 3, we need only prove (III) for $\mathcal{F}$. Since $Y \subset X \in \mathcal{F}$ means $Y \subset X \subset \subset E$. If $|X \backslash Y|=1$, then evidently, $Y \cup x=X \in \mathcal{F}$ for $x \in X \backslash Y$. If $|X \backslash Y| \geq 2$ and for any $x \in X \backslash Y, Y \cup x \notin \mathcal{F}$. Suppose there exists $Z \in \mathcal{F}, Y \subset Z \subset X$. Then by the inductive, there is $x \in Z \backslash Y \subset X \backslash Y$ such that $Y \cup x \in \mathcal{F}$, a contradiction. So, $Y$ is a maximal one strictly contained in $X$. Let $E \backslash Y=A, E \backslash X=B$. Then $B \subset A$ and $B$ is a closed set which is strictly contained in $A$. However, by Lemma 3, there is $C_{A} \subset A \backslash B$ such that $A \backslash C_{A}$ is a closed set of $(E, \tau)$. That is to say, $B \subset\left(A \backslash C_{A}\right) \subset A$, further, $E \backslash B \supset E \backslash\left(A \backslash C_{A}\right) \supset E \backslash A$, i.e. $X \supset\left(Y \cup C_{A}\right) \supset Y$ and $Y \cup C_{A} \in \mathcal{F}$, a contradiction.

Therefore, for $X, Y \in \mathcal{F}, Y \subset X$ holds which implies that there exists $x \in X \backslash Y$ satisfying $Y \cup x \in \mathcal{F}$.

Lemma 5 Let $(E, \tau)$ be an infinite convex geometry, and let $\mathcal{N}$ be the family of closed sets of $(E, \tau)$ and $\mathcal{F}$ the complement of $\mathcal{N}$. Then $(E, \mathcal{F})$ is an infinite antimatroid.

Proof Since $(E, \tau)$ satisfies $(\mathrm{AC})$ and $\mathcal{F}$ is the complement of $\mathcal{N}$, one easily finds that $\mathcal{F}$ satisfies (III) in Definition 3. Moreover, by Lemma 3, $\mathcal{F}$ satisfies (I) the conditions and (II) in Definition 3. Hence the needed result is obtained.

Theorem 2 Let $\mathcal{F}$ be a set system and $\mathcal{N}$ the complement of $\mathcal{F}$. Let $\tau$ be the relative operator to $\mathcal{N}$. Then the following statements are true.

(1) $(E, \mathcal{F})$ is an infinite antimatroid if and only if $(E, \tau)$ is an infinite convex geometry.

(2) If $\mathcal{F}$ satisfies that $X \in \mathcal{F}$ which implies $X \subset \subset E$. Then $(E, \mathcal{F})$ is an infinite antimatroid if and only if $(E, \tau)$ is a convex geometry. 


\section{Proof}

(1) It is straightforward from Lemma 2 and Lemma 5.

(2) Routine verification from Lemma 2, Lemma 4, Remark 2 and Corollary 2.

\section{Acknowledgements}

This research is granted by National Nature Science Foundation of China (61572011).

\section{References}

[1] Welsh, D.J.A. Matroid Theory. London: Academic Press Inc. 1976.

[2] Oxley, J. Infinite Matroids. In: White, N. (Ed.). Matroid Application. Cambridge: Cambridge University Press, 1992, pp.73-90.

[3] Mao, H. and Liu, S. Remarks on external elements in independence spaces. Southeast Asian Bulletin of Mathematics. 2005. 29: 939-944.

[4] Mao, H. and Wang, G. Rank function acts on characterization of an independence space to have a pair of disjoint bases. Acta Math. Sinica (Chinese series). 2010. 53(4): 717-720. (in Chinese)

[5] Mao, H. On posets and independence space, Journal of the Egyptian Mathematics Society. 2014. 22(1): 1-5.

[6] Wang, G. and Mao, H. Geometric lattices and independence spaces, Ease-West Journal of Mathematics. 2010. 12(1): 1-7.

[7] Mao, H. and Wang, G. Sub-independence-spaces. Chinese Quarterly Journal of Mathematics. 2010. 25(2): 293-299.

[8] Kort, B., Lovász, L. and Schrader, R. Greedoids. Heidelberg: Springer-Verlag Berlin. 1991.

[9] Björner, A. and Ziegler, G.M. Introduction to Greedoids. In: White, N. (Ed.) Matroid Application. Cambridge: Cambridge University Press. 1992, pp.284-357.

[10] Mao, H. On the maps between antimatroids. Northeast Mathematics Journal. 2006: 22(1): $55-66$.

[11] Mao, H. Some characterization of infinite antimatroids. Analele Stiintifice ale Universitatii Ovidius Constanta, Seria Matematica. 2009. 17(2): 115-122.

[12] Armstrong, D. The sorting order on a Coxeter group. Journal of Combinatroics Theory, Series A. 2009: 116(8): 1285-1305.

[13] Wahl, N. Antimatroids of finite character. Jouranl of Geometry. 2001. 70: 168-175.

[14] Mao, H. and Liu, S. On antimatroids of infinite character. Mathematica Pannonica. 2012. 23(2): 257-266. 
[15] Mao, H. Poset properties of infinite antimatroids. East-West Journal of Mathematics. 2011. 13(1): 51-56.

[16] Mao, H. Two characterizations of antimatroids. Annals of the Alexandru Ioan Cuza University-Mathematics. 2013. 59(2): 453-466.

[17] Grätzer, G. General Lattice Theory, 2nd. ed. Basel: Birkhäuser Verlag. 1998. 